\title{
ALGUNOS ASPECTOS DE LA ORGANIZACIÓN DE LA ASISTENCIA SANITARIA EN LA MALLORCA RURAL, SIGLOS XVIII Y XIX ${ }^{1}$ PUBLIC HEALTH ORGANIZATION IN RURAL MAJORCA (SPAIN), $18^{\text {th }}$-19 $9^{\text {th }}$ CENTURIES ALGUNS ASPETOS DA ORGANIZAÇÃO DA ASSISTÊNCIA SANITÁRIA NA MAIORCA RURAL (ESPANHA) NOS SÉCULOS XVIII E XIX
}

\author{
Isabel Moll Blanes ${ }^{2}$
}

\begin{abstract}
${ }^{1}$ Esta comunicación procede del trabajo realizado em el marco del proyecto de investigación "La accción de la Administración y de la Iglesia em el proceso de dotación de servicios públicos em España (1845-1930): un análisis regional”. Dirección General de la Investigación Centífica y Técnica, Ministerio de Educación y Ciencia (BHA2002-03783). Uma primera versión de este texto se presentó como comunicación em el X Simposio de Historia Económica, Universitad Autónoma de Barcelona, enero 2005.

2 Doctora en Ciencias Políticas. Catedrática de Historia Contemporánea del Departamento de Ciencias Históricas de la Universidad de las Islas Baleares - España.
\end{abstract}

PALABRAS CLAVE: Salud RESUMEN: El objetivo de este trabajo consiste en mostrar la existencia, al menos desde mediados del pública. Población rural. ochocientos, de dos redes de asistencia sanitaria en el mundo rural mallorquín. Una formada por Historia. profesionales sanitarios como médicos y farmacéuticos, encuadrados en el marco de la administración local. Otra formada por religiosas pertenecientes a congregaciones femeninas dedicadas a la educación infantil y juvenil, asi como al cuidado de enfermos. La documentación procede por lo que se refiere a los médicos, de listas aparecidas en el Semanario Económico de Mallorca (1779-1820) y en el Boletín Oficial de la Provincia (desde 1834); por lo que se refiere a la población religiosa a fondos procedentes del Archivo Diocesano de Mallorca y de los Archivos de los respectivos conventos.

KEYWORDS: Public health. Rural population. History.
ABSTRACT: The main issue of the paper is to describe the existence, from mid 19th century onwards, of two public health networks in Rural Majorca. The first deals with the set of health practitioners (physicians and pharmacists) engaged by local governments in order to give services to the population. A female religious population, dedicated to female education, and to health activity towards the poor and the population of the villages, form the second network. Data comes from official lists published in the Government Bulletins, and sources from Bishop Archive, as well as Convent Archives.
PALAVRAS-CHAVE: Saúde pública. População rural. História.
RESUMO: O objetivo deste trabalho consiste em mostrar a existência, pelo menos desde meados do século 19, de duas redes de assistência sanitária no mundo rural de Mallorca. Uma formada por profissionais sanitários como médicos e farmacêuticos, vinculados aos serviços governamentais e outra formada por religiosas pertencentes a congregações femininas dedicadas a educação infantil e juvenil, bem como ao cuidado dos doentes. A documentação relativa aos médicos, é originária de listas publicadas no "Semanário Econômico de Mallorca (1779-1820) e no "Boletín Oficial de la Província" (desde 1834); já a documentação relativa a população religiosa é procedente do Arquivo Diocesano de Mallorca e dos arquivos dos respectivos conventos.
Endereço: Isabel Moll Blanes

Carretera Valldemossa, s/n., km. 7,5

07121 Palma de Mallorca, Espanha.

E-mail: dhaimb0@uib.es
Artigo original: Pesquisa

Recebido em: 20 de junho de 2005

Aprovação final: 16 de novembro de 2005

Texto Contexto Enferm, Florianópolis, 2005 Out-Dez; 14(4):469-79. 


\section{INTRODUCCIÓN}

Una de las definiciones más claras y más útiles del concepto Salud Pública la proporcionó Ch. Winslow en 1920. Para este autor, por Salud Pública podemos entender " $[\ldots]$ the science and the art of preventing disease, prolonging life, and promoting physical health and efficiency through organized community efforts for the sanitation of the environment, the control of community infections, the education of the individual in principles of personal hygiene, the organization of medical and nursing services for the early diagnosis and preventive treatment of disease, and the development of the social machinery which will ensure to every individual in the community a standard of living adequate for the maintenance of health". 1:24 El presente texto enfoca una de las actividades que integran la definición precedente: la organización de los servicios de asistencia sanitaria en la Mallorca rural de la segunda mitad del ochocientos. Para ello se tratarán dos procesos que determinan la configuración de dicha asistencia: el proceso de creación de los servicios médicos por parte de la Administración local y el proceso de creación de una red de instituciones religiosas femeninas con una total dedicación a la acción social.

$\mathrm{El}$ primer proceso se encuentra fuertemente vinculado a la acción política local y estatal y sus protagonistas son los colectivos de profesionales de la sanidad (especialmente médicos y farmacéuticos) establecidos en el ámbito rural mallorquín; su relación con los Ayuntamientos, en un principio, es por medio de contrato con el fin de proporcionar asistencia a quienes no contaran con recursos suficientes, aunque desde mediados de siglo XIX se empieza a diseñar un marco legal que irá incorporando a estos sanitarios en el aparato de la administración local. El segundo se forma de manera aparentemente más espontánea (aunque con el respaldo institucional de la Iglesia) y está constituida por una serie de congregaciones religiosas femeninas que abren conventos en todos los pueblos de la isla para, entre otras cosas, dedicarse a la educación de la infancia (especialmente de las niñas) y al cuidado de enfermos y necesitados, lo que permite atribuir a estas religiosas el origen de las prácticas de enfermería en Mallorca, mucho antes de que esta actividad fuera considerada como una profesión.

Este doble proceso tiene lugar en España durante un período de cambios en la organización política, en el sistema social, y en la estructura de la economía que van a condicionar las formas de desarrollo de los dos procesos citados. De esta serie de cambios interesa resaltar, a guisa de introducción, dos series de datos. De una parte, las características del proceso de crecimiento de la población insular a lo largo del siglo XIX. De la otra las reformas legales en el sector de la administración pública española durante el mismo período. La razón de su inclusión radica en el hecho de considerarlas como las variables que, de forma más inmediata, van a influir en la formación de las citadas redes.

Entre 1787 y 1910 la población mallorquina pasa de tener 135.906 habitantes (de los que 31.965 se concentraban en la ciudad de Palma), a 257.015 en 1910 (67.544 en Palma). Considerando la tasa de crecimiento aritmético referida a períodos intercensales (Tabla 1) se observa como la tasa de crecimiento anual de la ciudad es superior a la de la Part Forana, donde incluso hay un decenio de fuerte pérdida de población (debido sobre todo a las migraciones ocasionadas por la crisis finisecular); aunque la serie muestra un crecimiento bajo y lento, atribuible a las bajas tasas de natalidad y mortalidad que se daban en la zona durante este período, de las que eran conscientes los mismos coetáneos.

Tabla 1 - Tasa de crecimiento anual de 1a población, 1787-1920*.

\begin{tabular}{cccc}
\hline Períodos & Palma & Mallorca & $\begin{array}{c}\text { Mallorca sin } \\
\text { Palma }\end{array}$ \\
\hline $1787-1838$ & 0,5 & & 0,4 \\
$1838-1860$ & 1,2 & & 1,1 \\
$1860-1877$ & 0,6 & & \\
$1877-1887$ & 0,5 & 0,7 & 0,8 \\
$1887-1897$ & 0,3 & $-2,4$ & $-3,2$ \\
$1897-1900$ & 0,0 & 0,3 & 0,5 \\
$1900-1910$ & 0,6 & 0,3 & 0,3 \\
$1910-1920$ & 1,4 & 0,5 & 0,2 \\
\hline
\end{tabular}

* Elaboración propia a partir de datos censales.

La serie muestra un crecimiento bajo y lento, atribuible a las bajas tasas de natalidad y mortalidad de la región, que ofrece, en su conjunto, notables diferencias con el resto de España. Así los estudios sobre la transición demográfica en España indican que la tasa de mortalidad (y en especial la infantil) de Baleares es una de las tasas más bajas de todo el país, al menos por lo que concierne al período de 60 años que va de 
1870 a $1930,^{2-5}$ tasas que no sólo corresponden a la provincia, sino también a su capital. El interés del tema no radica sólo en la constatación del fenómeno por parte de los historiadores, sino que los propios coetáneos eran conscientes de la situación. El ingeniero de caminos Eusebio Estada, en su estudio sobre la ciudad de Palma realizado en 1885, incluye datos demográficos correspondientes a los períodos 18611870 y 1878-1884; la comparación de los datos de Palma, Baleares y España muestra, al menos, dos cosas: 1) por lo que concierne a la mortalidad los datos señalan una mortalidad más elevada en Palma que en el resto de la provincia, un $27,1 \%$, frente a un $22,6 \%$, aunque en relación a la media española (un 30,7\%) las tasas son más bajas; 2)por lo que concierne a la natalidad, la situación cambia. La media provincial sin contar con la ciudad es de un 31,2\%, frente al 28,2 de Palma, mientras que la media española es de un 37,3\%". 6:214-5

Se observan, por tanto, unas tendencias demográficas distintas en población de la ciudad de Palma (la capital de la isla) y en la población rural. Este último espacio es el que configura el interés de este trabajo, de ahí que se considere necesario desagregar los datos precedentes con el fin de mostrar algunas consecuencias del crecimiento demográfico en la distribución espacial de la población rural, con dos referencias específicas; a saber: el aumento del tamaño de los núcleos urbanos y el aumento del número de municipios. En la Tabla 2A se ve como en 1787 el $26,5 \%$ de la población vivía en pueblos de más de 5.000 habitantes, mientras que en 1910 la proporción era del 47,5\%; en esta últimaa fecha, además, se produce un incremento de núcleos de más de 10.000 habitantes que concentran el 12\% de la población llamémosle rural. Este incremento hay que atribuirlo a pautas de diferenciación económica, como la consolidación de procesos de industrialización (calzado y textil, básicamente) o el desarrollo de actividades comerciales (en especial, exportación de productos agrícolas).

Tabla 2A - Total de población por tamaño de los pueblos, 1787 y 1910*.

\begin{tabular}{ccccc}
\hline & \multicolumn{2}{c}{1787} & \multicolumn{2}{c}{1910} \\
\hline Habitantes & Total & \% & Total & \% \\
\hline$<1.000$ & 2.910 & 2,8 & 3.172 & 1,7 \\
$1.000-1.999$ & 9.251 & 8,9 & 11.975 & 6,3 \\
$2.000-2.999$ & 26.505 & 25,5 & 26.908 & 14,2 \\
$3.000-4.999$ & 37.731 & 36,3 & 57.430 & 30,3 \\
$5.000-9.999$ & 27.544 & 26,5 & 66.522 & 35,1 \\
$>10.000$ & 0 & 0 & 23.464 & 12,4 \\
\hline
\end{tabular}

*Elaboración propia a partir de datos censales.

Otro hecho a resaltar en relación al crecimiento de la población, es el aumento del número de municipios. En la Tabla 2B se observa como se pasa de 34 municipios en 1787, a 48 en 1910; en consecuencia, un mayor número de núcleos de población con Ayuntamiento propio, lo que supone una mayor oferta potencial de los servicios derivados de las atribuciones en materia social que van recibiendo los municipios a lo largo del siglo XIX. Aunque esta última ventaja no siempre se traduce en la realidad dado que los municipios, especialmente los de nueva creación que solían ser los de menor tamaño, podían hacer frente a sus obligaciones legales debido a la continua falta de recursos de sus respectivos Ayuntamientos.

Tabla 2B - Municipios y su población, 1787 y 1910*.

\begin{tabular}{lcccccc}
\hline & $\mathbf{N}^{\mathbf{*}}$ & \multicolumn{2}{c}{ Habitantes } & \multicolumn{2}{c}{$\mathbf{N}^{\mathbf{*}}$} & \multicolumn{2}{c}{ Habitantes } \\
\hline Mun. & Total & $\mathbf{0}$ & Mun. & Total & $\mathbf{\%}$ \\
Manacor & 13 & 36.729 & 35 & 18 & 71.280 & 38 \\
Palma & 9 & 33.869 & 33 & 13 & 64.651 & 34 \\
TOTAL & 12 & 33.343 & 32 & 17 & 53.540 & 28 \\
\hline
\end{tabular}

*Elaboración propia a partir de datos censales. 
La afirmación precedente nos introduce en el segundo tipo de cambios que queremos señalar y que tienen como punto de referencia el marco políticolegal. Dentro de este marco podemos distinguir dos niveles normativos. Uno, derivado de la opción por una administración centralizada, se traduce en una nueva organización territorial que incorpora la legislación para constituir las Diputaciones provinciales y los Ayuntamientos de base representativa; ${ }^{7}$ de este nivel proceden tanto las leyes generales de Ayuntamientos, como cuestiones más específicas como sería la posibilidad de crear nuevos municipios a partir, por ejemplo, de procesos de segregación. Otro, desarrollado sobre todo a partir del Bienio Progresista (con raíces tanto en el último período ilustrado, como en los planteamientos políticos de la primera etapa constitucional), se refiere a la legislación específica de la organización de la Sanidad y de la Educación públicas, de la que se derivan las competencias provinciales y municipales sobre este tipo de actividades; por ejemplo, la creación de partidos médicos, la consolidación de los médicos titulares, la regulación de la formación de maestros, o el desarrollo del proceso de escolarización para ambos sexos que traduce experiencias muy diversas; todo un conjunto de actuaciones en las que los Ayuntamientos van a tener un importante protagonismo.

\section{LA ORGANIZACIÓN DE LOS SERVICIOS SANITARIOS}

\section{Médicos y farmacéuticos, 1795 - 1899}

Los aspectos a tratar sobre el proceso de formación y consolidación de la red de médicos rurales son los siguientes. En primer lugar, la relación del número de profesionales de la salud, médicos y farmacéuticos, especialmente, establecidos en los núcleos urbanos de los municipios; el primer conjunto de datos de que disponemos procede de 1795 y el último a 1899. En segundo lugar, la comprensión de esta red como resultado de una doble acción: una, específica de los ayuntamientos, que se remonta como mínimo - a mediados del siglo XVIII; otra, iniciada en torno a 1850, tiene su origen en el conjunto de leyes, órdenes y decretos promulgados por el Estado. En tercer lugar, la existencia de un proceso de diferenciación entre los profesionales de la sanidad, del que se tiene noticia desde finales del setecientos. Por último, considerar la relevancia de otros dos procesos: la profesionalización en el marco de instituciones académicas; y los cambios en el marco científico que se producen a finales del siglo XIX.

Las únicas series de datos sobre el total de profesionales de la sanidad existentes en Mallorca, no surgen de la misma fuente. Contamos con una lista procedente de un Acta del Real Acuerdo sobre arreglo de médicos que trata de la necesidad de permitir el ejercicio de la medicina, ${ }^{8}$ sólo a los profesionales con certificado de la Academia Médico-Práctica de Mallorca (ver Apéndice 1); de acuerdo con esta lista en 1795 había en Mallorca 87 médicos, 63 en Palma y 63 en el resto de la Isla, todos ellos habilitados por la Academia de Medicina. ${ }^{9}$ La segunda lista procede del Repartimiento Individual de la Contribución, de 1851. La tercera corresponde al listado de miembros del Colegio Oficial de Médicos y Farmacéuticos.

Se trata de listas cuyo interés radica en la información que proporcionan, al mostrar la distribución espacial de sanitarios en la isla. No son homogéneas en la medida que las profesiones relacionadas no son las mismas en cada una de las listas. Así, la lista de 1795 no especifica la profesión de los individuos, sólo que son individuos autorizados por la Academia Médico-Práctica para ejercer la medicina. La lista de 1852 es algo más compleja al incluir cuatro profesiones sanitarias distintas: médicos, médicoscirujanos, cirujanos (barberos, romanistas...) y farmacéuticos. Por último, la lista de 1899 es, posiblemente, la que presenta una mayor homogeneidad, ya que todos los profesionales reseñados se han formado en Facultades de Medicina y Farmacia y son miembros del Colegio de Médicos y Farmacéuticos, establecidos más o menos en la misma fecha. Con estas aclaraciones previas, consideramos que las listas proporcionan una información que permite valorar la existencia de una red de profesiones sanitarias en la Mallorca decimonónica que cubre la práctica totalidad del territorio insular. En la Tabla 3 se presentan datos agregados, relacionando el total de profesionales por partidos judiciales; de acuerdo con los datos individualizados por municipio se puede decir que prácticamente todos los pueblos de Mallorca contaban con un médico y la mayoría con un farmacéutico, al menos desde 1852. 
Tabla 3 - Total profesionales de la sanidad por partidos judiciales, 1795-1899*.

\begin{tabular}{lccc}
\hline PJ & $\mathbf{1 7 9 5}$ & $\mathbf{1 8 5 2}$ & $\mathbf{1 8 9 9}$ \\
\hline Inca & 26 & 46 & 50 \\
Manacor & 19 & 37 & 40 \\
Palma** & 18 & 26 & 34 \\
\hline TOTAL & 63 & 109 & 124 \\
\hline
\end{tabular}

* Elaboración a partir de los datos procedentes de los documentos citados más arriba.

**Sólo el municipio, se excluye la ciudad.

Las diferencias que se muestran entre 1852 y 1899 , obedecen a la inclusión en la primera lista de profesiones médicas que ya no se contemplan como tales en el período en que se confecciona la segunda. Ello se debe, como se comentará seguidamente, a un proceso progresivo de diferenciación en la línea de promocionar el ejercicio de la profesión sólo para quienes se hubieran formado en Facultades de Medicina.

Para entender el amplio espectro de la red y, en consecuencia, considerar sus efectos positivos sobre la población, conviene tener en cuenta las formas de intervención pública que hicieron posible la existencia de dicha red. No es un error pensar que hasta probablemente 1854 el Estado no se implicó de manera directa en poner los medios para dotar de este tipo de servicios a la población. Un Real Decreto de 5 de abril de dicho año determinaba que todas las ciudades, villas y lugares del reino, tendrán médicos, cirujanos y farmacéuticos titulares para la asistencia de los pobres, para el Socorro de las demás personas que necesitaren de su auxilio, y para el desempeño de otros deberes que se expresan en el lugar correspondiente. Ello no implicaba que con anterioridad a dicho Decreto no se contara con médicos en las poblaciones, sino que este servicio estaba bien a cargo de los Ayuntamientos, bien lo dispensaban voluntariamente los propios médicos. De hecho al menos desde el siglo XVI hay datos de la existencia de este servicio y la información que recoge el Apéndice 1 que se incluye al final de la comunicación es bastante explícito al respecto; la responsabilidad de los Ayuntamientos, a pesar de algunos intentos intervencionistas por parte de la Administración central presentes desde la Constitución de 1812, constituye la norma hasta el Decreto de 1854. A partir de este período la organización de los "partidos médicos" primero y de los médicos titulares más tarde, se irá estableciendo a lo largo de la segunda mitad del siglo XIX: primero por la Ley General de Sanidad de 1855 y luego por un Reglamento de 9 de noviembre de 1864 (nunca aplicado), otro de 11 de noviembre de 1868 que pudo aplicarse gracias a un Decreto de 24 de octubre de 1873 y, por último, un Real Decreto de 14 de junio de 1891. Esta línea de intervención de la Administración no prohibía, por supuesto, el libre ejercicio de la medicina, sino que ayudó a formar una red de profesionales que en Mallorca a finales del siglo XIX, cubría toda la isla.

La consolidación de la red de profesionales sanitarios se reforzó, a su vez, gracias a un proceso de profesionalización de la actividad médico-sanitaria determinado por su regulación por parte de las autoridades y por la formación recibida, todo ello en el contexto del significado de la asistencia médica antes y después de la transición sanitaria. En relación a la primera ya a finales del siglo XVIII (ver Apéndice 2) se observa una preocupación por parte de las autoridades locales de los problemas planteados por la cantidad de "impostores" actuando como médicos por lo que pusieron en marcha una serie de medidas para frenar este tipo de actividad. Posiblemente la más importante fue la de exigir el certificado de la Academia Médico Práctica de Mallorca "habilitando" al facultativo para ejercer su profesión; con esta medida los Alcaldes de los pueblos sólo debían aceptar a quienes presentaran dicho certificado. Con la Ley General de Sanidad se reforzó la profesión médica ya que uno de sus artículos especificaba que sólo podían ejercer la medicina y la cirugía quienes ostentaran títulos de Facultades y Escuelas. Por último la creación de los Colegios de Médicos y Farmacéuticos a finales del ochocientos completó la exclusividad de la profesión.

En relación a la segunda sólo podemos proporcionar algunas consideraciones puntuales. En 1824 se cerró la Facultad de Medicina de la Universidad Literaria de Mallorca (y unos años más tarde se cerró dicha Universidad), lo que supuso que la formación y la información que recibieron quienes optaron por estudiar cualquier carrera relacionada con la sanidad (medicina, cirugía, farmacia) lo hicieron de centros como las Universidades de Valencia, Granada, Montpellier, Barcelona y en unos pocos casos, París; ello pudo significar la vía para introducir los avances científicos en medicina. Para poder evaluar la relevancia de estas mejoras contamos con algunas referencias como la Revista Balear de Ciencias Médicas (1885), los discursos presentados en las sesiones de apertura de la Real Academia de Medicina (1786) o del Colegio MédicoFarmacéutico (1899). También acontecimientos más puntuales como la apertura en Palma en 1896 de un 
Instituto de Vacunación directa y de un Laboratorio Químico Biológico. Se trata de indicaciones que todavía no están lo suficientemente documentadas para poder ofrecer algún tipo de explicación acerca de la efectividad de las mejoras sobre la población. Pero, como ejemplo de por donde se puede seguir una línea de investigación sobre este tipo de temas, queremos citar un extracto del Discurso que el doctor Tomás Darder leyó en la sesión inaugural de la Real Academia de Medicina y Cirugía de Palma en enero de 1901: durante el último tercio del siglo XIX Mallorca ha seguido, en todos sus puntos, la magestuosa trayectoria trazada por el progreso de la medicina. Auqnue careciendo de luz propia; como los planetas, ha reflejado siempre con viva intensidad la que, desde los centros más populosos, irradiaron los astros de primera magnitud, a través de los espacios infinitos de la ciencia. ${ }^{10}$

\section{Los servicios de enfermería: las congre- gaciones religiosas femeninas}

La formación de este tipo de redes de asistencia pública fue un fenómeno de cierta amplitud en Europa, al menos desde mediados del siglo XVII. Pero no se puede hablar de un modelo único de difusión de este tipo de asistencia social, ni tampoco de pautas que fijaran de manera específica donde y cuando debían instalarse. Desde este punto de vista vemos la posibilidad de proponer dos planteamientos metodológicos: considerar la creación de estas redes como una respuesta a necesidades específicas; y utilizar estudios de casos como una cuestión de método, teniendo en cuenta su significado y su representatividad.

Además, y en el caso concreto que nos ocupa, creemos que la creación de esta red no puede entenderse como un fenómeno aislado, sino como respuesta a situaciones que pedían este tipo de intervención, lo cual implica supone considerar los temas siguientes:

1) la naturaleza de las estructuras de poder que comprendía:
- tensiones entre la Iglesia y el Estado desde fines del siglo XVIII; especialmente importantes de 1833 en adelante. La fueente de estas tensiones: el progresivo control de la educación y de la beneficencia por parte del Estado, en un contexto de secularización de la política estatal que suponía la pérdida de poder por parte de la Iglesia;

- tensiones en el seno de la población en relación con el modelo que se optó del nuevo Estado, tanto en el aspecto ideológico, como en el de la organización territorial;

2) el tipo de estructura social del que proceden $y$ en el que actúan este tipo de instituciones, y los problemas que surgen como resultado de los cambios que se produjeron en el seno de dicha estructura. Además de los apuntados más arriba cabría considerar:

- cambios en la organización del sector económico;

- modificaciones en la estructura social;

3) el reforzamiento de algunas pautas culturales, como la filantropía y la mentalidad cristiana que mediatizaron tanto las decisiones de crear nuevas congregaciones, como las decisiones de las mujeres a dedicar sus vidas a este tipo de actividades.

Desde la Edad Media había en Mallorca un importante colectivo - masculino y femenino - viviendo en Conventos y Monasterios. A finales del XVIII el $74 \%$ de las instituciones religiosas se encontraban en Palma, el origen del 95\% de las órdenes era de fuera de Mallorca y el 94\% de los religiosos eran hombres. En cuanto a su naturaleza, la práctica totalidad de los centros femeninos era de clausura y su función primordial era la oración. Todo ello cambiará a lo largo del XIX (Tabla 4) con la aparición de nuevas congregaciones religiosas que pronto formarán una densa red generadora de servicios como educación, asistencia a enfermos y a cuidar a la población necesitada.

Tabla 4 - Distribución de los Conventos por género y localización*.

\begin{tabular}{ccccc}
\hline & Palma & Part forana & Masculinos & Femeninos \\
\hline 1787 & $74 \%$ & $26 \%$ & $64 \%$ & $36 \%$ \\
1870 & $13 \%$ & $87 \%$ & $33 \%$ & $67 \%$ \\
\hline
\end{tabular}

* Los datos de 1787 corresponden a la información proporcionada en el Censo de Floridablanca; los de 1870, al Censo Oficial del mismo año 
Para esta trabajo interesa el análisis de las congregaciones femeninas, ya que en términos de asistencia social son quienes van a tener el mayor protagonismo. El período en que se crean estos centros abarca de 1798 (cuando el sacerdote Antonio Roig funda en Felanitx la Congregación de las Hijas de la Caridad) a 1915 (cuando finaliza el pontificado de Pere Joan Campins, quien creará el Instituto Diocesano de Caridad para ordenar y organizar la distribución y la actuación de estas congregaciones); aunque ello no signifique el fin de la expansión de estas congregaciones, ya que va a continuar durante algunos años más. En este período se abren 104 centros que proceden de 14 congregaciones religiosas femeninas, fundadas por mallorquines y formadas por personal autóctono; y también de la instalación en la isla de 3 congregaciones externas dedicadas única y exclusivamente a la educación de las hijas de las familias adineradas de Mallorca (dos se implantan en Palma o en sus alrededores y una en Sóller). Las diferencias entre estas nuevas fundaciones y las antiguas órdenes religiosas proceden de la normativa que las rige (son congregaciones, no órdenes), y la finalidad de su acción (participación activa en la vida social del lugar donde se localiza el convento, especialmente en el sector benéfico y educativo).

El número de Congregaciones en 1915 1(104) es el resultado de un proceso previo de fusión iniciado en 1880: de las 14 congregaciones existentes en 1870, se pasó a 11 en 1915. Estas fusiones, organizadas desde la dióceses con el fin ordenar la dispersión a la que se había llegado en la fecha indicada, permitieron la consolidación de tres congregaciones que controlaban el $88 \%$ del total de conventos femeninos en Mallorca. Eran las Hijas de la Misericordia, las Hijas de la Caridad y las Agustinas (Tabla 5), cuyos estatutos eran muy similares, coincidiendo siempre con los mismos objetivos: enseñanza (especialmente infantil y femenina), atención a pobres, enfermos y necesitados.

Tabla 5 - Número de Conventos por Congregación, 1915.

\begin{tabular}{lcccc}
\hline & Palma & $\begin{array}{c}\text { Part } \\
\text { Forana }\end{array}$ & TOTAL & $\%$ \\
\hline Hijas de la Misericordia & 4 & 35 & 39 & 38 \\
Hijas de la Caridad & 10 & 24 & 34 & 32 \\
Agustinas & 8 & 11 & 19 & 18 \\
Trinitarias & 1 & 2 & 3 & \\
$\begin{array}{l}\text { Hermanas de la } \\
\text { Providencia }\end{array}$ & 1 & 1 & 2 & \\
$\begin{array}{l}\text { Misioneras Sagrados } \\
\text { Corazones }\end{array}$ & 0 & 2 & 2 & 12 \\
Religiosas de la Pureza & 1 & 0 & 1 & \\
Otras & 3 & 1 & 4 & \\
\hline TOTAL & 28 & 76 & 104 & 100 \\
\hline
\end{tabular}

Como el proceso abarca un período bastante amplio - de 1798 a 1915 - hemos decidido fijar unos subperíodos en relación a los pontificados de los Obispos encargados de la Diócesis. De acuerdo con los datos de la Tabla 6 vemos como el $70 \%$ de los Conventos se abrieron entre 1851 (fecha del Concordato del Estado con la Santa Sede) a 1898; ello muestra que la expansión de la red en la Part Forana se consolidó en torno a 1880.

Tabla 6 - Pontificados y fundaciones, 1794-1915.

\begin{tabular}{clcc}
\hline Período & Pontificado & Fundaciones & Conventos \\
\hline $1794-1818$ & Bernat Nadal & 4 & 4 \\
$1819-1825$ & Pedro González & & \\
$1825-1842$ & Antonio Pérez de Hirias & & \\
$1847-1851$ & Rafael Manso & 1 & 3 \\
$1851-1873$ & Miquel Salvà & 4 & 34 \\
$1875-1886$ & Mateu Jaume & 2 & 23 \\
$1886-1897$ & Jacinto María Cervera & & 16 \\
$1898-1915$ & Pere Joan Campins & 2 & 24 \\
\hline
\end{tabular}

En relación a la responsabilidad de la expansión hay que considerar de manera separada la fundación de una Congregación y la apertura de un Convento. La Iglesia tuvo la máxima responsabilidad en dichas fundaciones, aunque no siempre fueren bien vistas por las autoridades eclesiásticas. De hecho hubo bastante oposición a que las mujeres intervinieran en la sociedad de la manera como se planteaban las reglas de las 
Congregaciones, ya que la función de las mujeres en el seno de la Iglesia era la vida contemplativa (la oración...) y no el compromiso social. Pero, ya se ha citado más arriba, bien por filantropía, bien por la necesidad de hacer frente al Estado en los mismos términos de intervención social, algunos sacerdotes financiaron las fundaciones que siempre debían tener la aceptación del Obispo; así un Obispo (Bernat Nadal a comienzos del ochocientos), siete sacerdotes, tres monjas y tres mujeres que después profesaron fueron quienes pusieron en marcha las Congregaciones. En cuanto a la apertura de conventos el espectro de responsabilidades es mucho más amplio: Ayuntamientos, médicos y grupos de trabajadores.

La última información sobre la naturaleza de las fundaciones se refiere al origen social y geográfico de las religiosas. Como se acaba de citar, la mayoría de fundadores son casi todos sacerdotes que también proceden del mundo rural; suelen ser hijos de pequeños propietarios campesinos, de grandes arrendatarios, e incluso alguno procede de familia de grandes terratenientes locales (don Gabriel Ribas de Pina, fundador de las Hermanas de la Misericordia). El personal reclutado -las religiosas- procede también del sector rural, y en su mayor parte del grupo de pequeños propietarios; hasta finales del siglo XIX éste va ser su "ámbito" de desarrollo natural. Pero cuando el crecimiento de Palma supera el recinto amurallado y empiezan a surgir pequeños núcleos de población que si bien son de extracción campesina se hallan en la zona de expansión urbana de la ciudad, el "modelo" de asistencia que tiene su origen en el mundo rural, empieza a asentarse en estos nuevos barrios; igual que el personal de las congregaciones. Este tipo de procedencia, social y geográfica, va a generar una serie de canales de información, al tiempo que permite entender la difusión y la penetración de nuevas pautas de higiene, salud y nutrición en una sociedad aparentemente hermética. Pero no sólo funcionó en esta línea la red de parentesco, sino que la asistencia de niñas a las escuelas de las monjas era prácticamente general, al menos desde principios del siglo XX. De ahí la necesidad por conocer las características del discurso educativo, igual que analizar el significado de la educación impartida en dichas escuelas como una vía para transmitir pautas de comportamiento específico que se introducían en el mismo contexto familiar, como pautas normativas a menudo atribuidas sólo a las mujeres.

Si se considera la localización de los centros por Congregación, en este caso nos ceñimos a las tres Congregaciones más importantes, podemos observar algunas diferencias interesantes (Tabla 7): las Hijass de la Caridad tenían una mayor presencia en Palma y las Hijas de la Misericorda en el mundo rural.

Tabla 7 - Localización de Conventos por Congregaciones, 1915.

\begin{tabular}{lcccc}
\hline & \multicolumn{2}{c}{ Palma } & \multicolumn{2}{c}{ Part Forana } \\
\hline & Total & $\mathbf{0}$ & Total & $\mathbf{0}$ \\
\hline Hijas de la Misericordia & 4 & 10 & 35 & 90 \\
Hijas de la Caridad & 10 & 29 & 24 & 71 \\
Agustinas & 8 & 42 & 11 & 58 \\
\hline
\end{tabular}

Pero si se relaciona la localización con el tamaño de las poblaciones (Tabla 8), vemos como el 60\% de los centros de las Hijas de la Misericordia y el 55\% de los de las Agustinas se encontraban en núcleos de menos de 2000 habitantes, mientras que el 71\% de los Conventos de las Hijas de la Caridad se localizaban en pueblos de más de 2.000 habitantes.

Tabla 8 - Distribución de Conventos por tamaño de la población Mallorca, 1910 (\%).

\begin{tabular}{lccc}
\hline & HM & HC & A \\
\hline$<1000$ & 26 & 8 & 18 \\
$1001-2000$ & 34 & 21 & 37 \\
$2001-3000$ & 14 & 17 & 9 \\
$3001-5000$ & 20 & 21 & 27 \\
$5001-8000$ & 0 & 8 & 9 \\
$>8000$ & 6 & 25 & 0 \\
& 100 & 100 & 100 \\
\hline
\end{tabular}

¿Nos encontramos frente a una estrategia de localización? Posiblemente sí, en especial si tenemos en cuenta la información que dábamos al comienzo de la comunicación, relativa al crecimiento de la población y a las reformas políticas, en especial la posibilidad de crear más municipios en el marco del desarrollo del Estado. Así en Mallorca se pasó de 33 municipios en 1787 a 46 en 1860 y era frecuente que sus Ayuntamientos no contaran con recursos suficientes para desarrollar las políticas que determinaban las sucesivas leyes municipales, por lo que las religiosas ofrecían - de manera gratuita (por amor a Dios) - este tipo de servicios. También hay que considerar el empeoramiento de las condiciones de vida en especial a raiz de la crisis agraria finisecular, y muy especialmente la necesidad de reforzar la posición de la Iglesia frente a la política del Estado en ámbitos de Beneficencia, Salud y Educación. 
El panorama diseñado por la fundación de las congregaciones femeninas muestra una red densa y tupida que abarca todo Mallorca, de la ciudad de Palma, a los núcleos de población más pequeños localizados en la zona rural. A finales del siglo XIX se puede decir que apenas hay núcleos de población que no cuenten con uno de estos centros, los cuales hay que entender en el marco del nivel de desarrollo de los sistemas de salud pública de la época. Estas religiosas vienen a ser como agentes sanitarios intermedios que aun careciendo de una buena formación en el ámbito de la enfermería, van a proporcionar una ayuda inestimable a la población mallorquina mediante diversas formas de actuación, entre las que cabe destacar el ser conductoras/canalizadoras del discurso higienista, sobre todo en el seno de la población femenina.

A partir de los datos expuestos se puede inferir que a finales del siglo XIX apenas había un pueblo o una aldea sin un médico, un farmacéutico o al menos un Convento y, por los datos que actualmente estamos manejando, de una comadrona (Tabla 9). Para comprender mejor el significado de este tipo de datos, en especial desde la perspectiva de las Instituciones religiosas, es preciso insertarlos en el contexto de los procesos de desarrollo de los sistemas de Salud Pública de su tiempo. Es cierto que el estudio de estas Instituciones presenta la doble faz del dios Jano. De una parte hay que entender su función como parte del "rearmamento" de la Iglesia frente al Estado liberal. Pero de la outra, especialmente si la consideramos desde un punto de vista actual, también se puede entender su función como agentes sanitarios intermedios que si bien no tenían una buena formación en enfermería (porque de hecho tampoco en este período se había generalizado este tipo de formación), proporcionaron una gran ayuda a la población de Mallorca por medio de diferentes vías, como por ejemplo, como un canal de difusión del discurso higienista sobre todo entre las mujeres, transformando lo que en un principio se entendía como una norma, en un valor específicamente femenino.

Tabla 9 - Los servicios sanitarios, 1860 y 1900.

\begin{tabular}{lcccccc}
\hline & $\mathbf{1 8 6 0}$ & & & $\mathbf{1 9 0 0}$ \\
\hline P.J.(1) & NU(2) & PS(3) & Conv. & NU & PS & Conv. \\
\hline Inca & 25 & 36 & 6 & 25 & 36 & 25 \\
Manacor & 16 & 27 & 11 & 16 & 23 & 25 \\
Palma & 23 & 21 & 5 & 23 & 26 & 20 \\
\hline TOTAL & 64 & 84 & 22 & 64 & 85 & 70 \\
\hline
\end{tabular}

(1) Partidos Judiciales; (2) Núcleos urbanos; (3) Profesionales Sanitarios.

\section{CONCLUSIONES}

Como resumen de todo lo dicho interesa señalar cuestiones a guisa de reflexión general referida a los objetivos del trabajo: 1) la relevancia de la existencia de estas formas de asistencia médica con anterioridad a la transición epidemiológica. Con independencia del tipo de medicina que practicaran, no se debe olvidar la prestación de servicios sanitarios a una población que no podía contar con el marco institucional que ofrecía una gran ciudad; 2) su significado en el contexto de confrontaciones ideológicas que a menudo distorsionan el enfoque del análisis, primando los aspectos ideológicos sobre los asistenciales; 3) considerar que si bien se puede pensar en la existencia de una colaboración entre ambos colectivos, no se puede obviar la posibilidad de conflictos internos derivados de enfrentamientos entre religiosas y médicos, entre médicos y Ayuntamientos, o entre población y médi$\cos$; 4) la necesidad de profundizar en cada uno de estos procesos, ya que la información proporcionada mediante este trabajo sólo muestra la existencia y localización de dichos servicios; 5) huir de los encuadramientos formales a la hora de realizar el estudio de estos procesos. Es decir, no creemos razonable dividir el estudio a partir de áreas de conocimiento específicas, sino que consideramos más necesario fijar con claridad la naturaleza del problema e intentar la asimilación de diferentes aproximaciones científicas con la finalidad de conseguir mejores resultados*. 


\section{REFERENCIAS}

1 Winslow CEA. The untilled fields of public health. Science 1920 Jan. 51; (1306): 23-3.

2 Dopico F. Desarrollo económico y social y mortalidad: diferencias regionales (1860-1950). Dynamis 1985/1986; 5/6: 381-99.

3 Dopico F. Reher, D. El declive de la mortalidad en España, 1860-1930. Zaragoza: Asociación de Demografía Histórica; 1998.

4 Domínguez R. El índice físico de calidad de vida en España: evolución de las disparidades espaciales, 1860-1910. In: Torres J, Yun B, organizadores. Consumo, condiciones de vida y comercialización: Cataluña y Castilla, siglos XVIIXIX. Valladolid: Junta de Castilla y León; 1999. p.183-204.

5 Bujosa F, Moll I, Sureda B. La avanzada transición demográfica en Mallorca: el caso de la mortalidad infantil. Boletín de la Asociación de Demografía Historia 2000; XVIII (II): 125-46.

6 Estada E. La Ciudad de Palma. 2a ed. Palma: Govern de les Illes Balears; 2003.

7 Castro C. La Revolución Liberal y los municipios españoles. Madrid: Alianza Universidad; 1979.

8 Moll I. Noticia de El Semanario Econômico: 1778-1820. Mayurqa 1980; 17: 63-70.

9 Bujosa-Homar, F. La Academia Médico-Práctica de Mallorca. Palma: Institut d' Estudis Baleàrics; 1979.

10 Enseñat, TD. Discursos leídos en la sesión inaugural celebrada por la Real Academia de Medicina y Cirujía de Palma, el dia 27 de Enero de 1901, Palma de Mallorca: Tipografía de las hijas de Juan Colomar; 1901.

\section{APÉNDICE 1}

Semanario Económico, 42, 17 Octubre 1795

Auto del Real Acuerdo sobre arreglo de Médicos

El Real Acuerdo de la Audiencia de Mallorca con auto de 17 de Agosto de este año de 1795 se ha servido mandar: "Que el Presidente de la Real Academia Médico-Práctica, forme lista de todos los Facultativos aprobados por la misma que pueden exercer su profesión y se entregue un exemplar en todas las Boticas con la prevención que no admitan receta alguna de Médico que no sea de los aprobados pena de 40 libras aplicadas por mitad a penas de Cámara y gastos de justicia, y en la misma pena incurrirá todo Médico que sin ser Socio exerza la Facultad".

En su consecuencia se ha impreso y repartido una lista de todos los Médicos habilitados que existen actualmente en esta Isla, cuyo total es de 87 á saber cada una de las siguientes poblaciones tiene:

\begin{tabular}{|l|c|l|c|l|c|}
\hline Palma & 24 & Alcudia & 1 & Artà & 3 \\
\hline Algaida & 2 & Alaró & 2 & Son Servera & 2 \\
\hline Andratx & 1 & Binissalem & 3 & Campos & 2 \\
\hline Bunyola & 1 & Campanet & 1 & Felanitx & 3 \\
\hline Calvià & 1 & Inca & 3 & San Joan & 1 \\
\hline Esporles & 1 & Sta.Margalida & 1 & Manacor & 3 \\
\hline Llucmajor & 5 & Muro & 2 & Montuiri & 1 \\
\hline Marratxí & 1 & Mancor & 1 & Petra & 3 \\
\hline Sta. Maria & 1 & Selva & 2 & Porreras & 1 \\
\hline Sóller & 3 & Pollença & 4 & & \\
\hline Fornalutx & 1 & Sa Pobla & 2 & & \\
\hline Valldemossa & 1 & Sineu & 3 & & \\
\hline & & Sencelles & 1 & & \\
\hline
\end{tabular}

\section{APÉNDICE 2}

Semanario Económico, 7, 17 de Febrero de 1798

\section{Vando que prohibe los Curanderos, y prescribe} reglas para los Cirujanos, Boticarios y otros

D. Antonio Cornél, Caballero Comendador de Oreja en la Orden de Santiago, Teniente General de los Reales Exercitos de S.M.\&c. Gobernador y Capitan General del Exercito y Reyno de Mallorca, \&., y Presidente de su Real Audiencia, Regente y Ohidores \& c.

Por quanto se observan muchos abusos perjuiciales á la salud pública, y cada dia se aumenta el número de Cirujanos, Sangradores, Barberos, Parteras ò comadres, Clerigos, Frailes, Charlatanes, y otros muchos que haciendo ilusorias las anteriores providencias dadas por el Gobierno, engañan con sus estafas á ignorantes incautos, que padecen algunos achaques, ordenando sangrias, purgas, xaraves, píldoras y otros remedios, que dados sin el debido conocimiento pueden causar los efectos más fatales, como efectivamente lo han acreditado repetidas victimas de semejante idiotismo. Vista la solicitud de la Real Academia Medico-Practica de esta Isla, que para evitar semejantes excesos, nos ha suplicado renovásemos los Bandos publicados para dicho fin, uno en 10 de Noviembre de 1724, y otro en 24 de Julio de 1736: Por tanto, por autos de 22 de Febrero de 1790, y de 24 de Setiembre de 1792, tuvimos á bien mandar renovar los referidos Bandos; y para que tenga su devido efecto lo por Nos provehido, ordenamos y mandamos, que en pena de 10 libras por la primera vez, de 25 por la segunda, y de cinqüenta libras por la tercera, aplicaderas, una tercera parte, á penas de Cámara y gastos de justicia, y las dos restantes á la 
misma Academia Medico-Practica: ninguna persona de qualquier grado ò condicion que sea, pueda exercer la Facultad de Medicina, ni mandar, ó hacer sangrias, receptar purgas, xaraves, pociones, píldoras, ni otros remedios sin tener la aprobación, y pase de la Academia para exercer dicha facultad, advirtiendo, que à mas de la pena expresada de cinqüenta libras, se suspenderà de oficio á los Cirujanos, Sangradores, Barberos y comadres, y á los Charlatanes y Curanderos, se les castigará, según las leyes del Reyno; y que asimismo ningun Boticario, bajo dichas penas, podrá despachar receta, ni droga alguna, sin firma de Medico aprobado; pues à este fin se les entregará una nómina de todos los Abilitados para la practica. Por ultimo, al Medico que por qualquier via, titulo ó razon, contribuyere á ocultar qualquier exceso de los comprendidos en este Bando, por la primera vez será multado con cinqüenta libras que tendrán igual destino al expresado; y por segunda, con suspensión de Oficio por dos años. Y para que llegue á noticia de todos, y nadie pueda alegar ignoracia, mandamos publicar el presente Bando, y que se fixe en los lugares acostumbrados de esta Ciudad, la de Alcudia, y demas Villas y Lugares forenses. Dado en la Sala del Real Acuerdo á 25 de Enero de 1798. = D. Antonio Cornél. $=D$. Tomás Saez de Parayuelo. $=D$. Tomás Quartero. $=D$. Nicolás Campaner Sastre de la Geneta. = Por mandado de su Excelencia. $=$ D. Onofre Gomila Notario Escribano. 